\title{
Symptoms, signs, suspicion and setting: a PESI score for cancer-associated pulmonary embolism?
}

\author{
Florian Posch ${ }^{1,2}$ and Cihan Ay ${ }^{1,3}$
}

Affiliations: ${ }^{1}$ Clinical Division of Haematology and Haemostaseology, Dept of Medicine I, Comprehensive Cancer Center Vienna, Medical University of Vienna, Vienna, Austria. ${ }^{2}$ Clinical Division of Oncology, Dept of Medicine, Comprehensive Cancer Center Graz, Medical University of Graz, Graz, Austria. ${ }^{3}$ Dept of Medicine, Thrombosis and Hemostasis Program, McAllister Heart Institute, University of North Carolina at Chapel Hill, Chapel Hill, NC, USA.

Correspondence: Cihan Ay, Medical University of Vienna, Dept of Medicine I, Clinical Division of Hematology and Hemostaseology, Waehringer Guertel 18-20, Vienna A-1090, Austria. E-mail: cihan.ayameduniwien.ac.at

@ERSpublications

Suspect it or not: a call for assessing the severity of cancer-associated pulmonary embolism http://ow.ly/VvAB3079LFZ

Cite this article as: Posch F, Ay C. Symptoms, signs, suspicion and setting: a PESI score for cancer-associated pulmonary embolism? Eur Respir J 2017; 49: 1602225 [https://doi.org/10.1183/13993003.02225-2016].

Pulmonary embolism (PE) is a frequent complication in patients with cancer [1]. In contrast to PE in non-cancer patients, a considerable proportion of those in the oncological setting is detected incidentally on routine computed tomography (CT) staging/restaging examinations in asymptomatic (or, in hindsight, often oligosymptomatic) patients [2]. Different terminologies for this phenomenon exist in the literature, including unsuspected, incidental, and asymptomatic PE, with asymptomatic PE being clearly not preferable as many patients do report symptoms in retrospect [3]. Managing these so-called unsuspected pulmonary embolisms (UPEs) is a major challenge, because the clinical implications of this ill-defined condition are not fully understood and optimal therapeutic strategies are unclear $[4,5]$. In this issue of the European Respiratory Journal, FonT et al. [6] report observational evidence from the Spanish ambispective EPIPHANY registry, which has some relevance to the emerging topic of UPE. This is one of the few studies specifically dedicated to cancer-associated PE [2], and the analysis included subject-level data on anticoagulation, recurrent thrombosis and death from more than 1000 patients with this condition, rendering it one of the largest studies to date in the field. The investigators identified distinct patterns of 30-day mortality depending on whether patients had PE that was clinically suspected or not, and did or did not present with symptoms and/or signs suggestive of $\mathrm{PE}$ at the time of routine CT imaging performed for reasons other than PE diagnosis. These patterns prompted the investigators to derive a new three-level empiric classification of cancer-associated PE, which distinguishes the three scenarios 1) suspected pulmonary embolism (SPE), 2) truly asymptomatic UPE (TA-UPE) and 3) UPE with symptoms (UPE-S). Two risk classification schemes for cancer-associated PE have already been developed [7, 8]. What is the added value of this one? Is this new classification relevant for clinical decision making? And should it be adopted in the management of patients with cancer-associated PE?

Let us exemplify the clinical dilemma with two scenarios in clinical practice. The first case is about a 65 -year-old female patient undergoing cytotoxic chemotherapy for metastatic gastric cancer, who is referred

Received: Nov 102016 | Accepted: Dec 122016

Support statement: The authors' research on thrombo-oncology is partly funded by the Austrian Science Fund (FWF-SFB-54 "InThro"). Funding information for this article has been deposited with the Open Funder Registry.

Conflict of interest: None declared.

Copyright CERS 2017 
to the emergency department due to new onset of pleuritic chest pain and dyspnoea. The treating physician immediately raises the suspicion of cancer-associated PE, confirms the diagnosis by CT pulmonary angiography, and informs the haemodynamically stable patient that anticoagulation for at least 6 months will help to stabilise this clot and prevent its recurrence, keeping the bleeding risk at an acceptable level. While this clinical vignette illustrates a "textbook" scenario for which some evidence is available to guide diagnostic and therapeutic management [9], the "real-world" clinical picture of cancer-associated PE is often different and much more complex. For example, a more common scenario from our clinical practice would be an out-of-hospital radiologist's office informing us via telephone that they just detected a segmental PE on our patient's routine restaging CT scan but the patient already went home and their current health status is unclear (although the administrative assistant at the radiologist's welcome desk said this patient looked relatively well upon leaving the radiology office). What should be done with such a patient? Should they be called by telephone and immediately transferred to the hospital? Or would it be sufficient to treat this patient in 2 days' time when they will come to the clinic for their scheduled chemotherapy appointment? Should this patient be anticoagulated with full weight-based dose like a patient with symptomatic lobar cancer-associated PE or may it even be possible to withhold anticoagulation? These rather provocative questions underline the tremendous amount of uncertainty in managing and treating this patient for their UPE. However, there is also some certainty in this clinical scenario. First, UPE accounts for up to $50 \%$ of PE diagnoses in cancer patients treated at specialised cancer centres [10]. Second, UPEs seem to have a similarly adverse prognostic impact on mortality and recurrence of thrombosis to SPEs [11]. Third, there is a gap in the available evidence for treatment of UPEs because this patient subgroup was excluded from even the most recent randomised controlled trial investigating the efficacy and safety of anticoagulant treatment with low molecular weight heparin versus warfarin for cancer-associated thrombosis [12]. Finally, guideline-endorsed risk assessment models that inform whether patients with PE should be hospitalised or may be treated as outpatients, such as the (simplified) Pulmonary Embolism Severity Index ((s)PESI) [13, 14], have limited utility for cancer patients, because they innately classify cancer patients in the "not low-risk" categories [15] or even fail to predict short-term mortality at all [16]. Therefore, cancer-specific PE risk tools have been developed [7, 8], but they do not account for UPE. Thus, contemporary clinical data that could fill this evidence gap are much needed to identify patients with cancer-associated PE who can safely be treated as outpatients $[17,18]$.

The EPIPHANY study provides some direction to specific PE risk assessment in patients with cancer. In this multicentric study, the clinical course of cancer-associated PE was investigated by examining the relationship between general and cancer-specific baseline variables, symptoms, anticoagulation, $\mathrm{PE}$ recurrence and 30-day mortality in more than 1000 patients. It is important to discuss the clinical relevance of the study in light of some major limitations first. Although a relatively large size, every second patient has been enrolled retrospectively. This is particularly concerning when considering that many of the study's major results are based on the analysis of symptoms, which are known to be hard to assess even in a prospective fashion and taking the complexity encountered in cancer patients into account. Therefore, some underreporting and misclassification of symptom status is likely. It is also not clear how the causes of death were adjudicated (probably not by autopsy) and the investigators used cause-of-death categories of questionable validity such as "cause of death from PE and other conditions". Another major limitation is that every fifth patient in EPIPHANY was a patient treated in the adjuvant setting of cancer therapy. These patients have a venous thromboembolism (VTE) risk profile more similar to non-cancer PE patients, with surgery performed with curative intent and the post-surgical period being the most important causal determinant of PE risk, followed by a rapid decline of PE risk after this phase. This is in stark contrast to the many medical oncology patients with cancer-associated PE having metastatic disease, where a thrombotic stimulus from the disseminated cancer is the major causal and also persisting determinant of PE risk [19]. Exclusion of these adjuvant cases may have led to a more meaningful patient population. A final limitation is the apparent underreporting of isolated subsegmental PE, which is known to be relatively common in UPE patients but was only reported in $1 \%$ of patients in EPIPHANY [20]. Nevertheless, these limitations should not hide the fact that EPIPHANY reveals six important findings.

1) $58 \%$ of PE diagnoses in EPIPHANY were made without initial suspicion of PE. This confirms UPE as a major subentity of cancer-associated PE.

2) Patients with SPE had more proximal clots, as well as a higher frequency of concomitant deep vein thrombosis. This demonstrates a positive association between clot burden and extent of symptoms at diagnosis of cancer-associated PE, thereby contradicting previous reports that did not find such an association [5].

3) The primary end-point of EPIPHANY, 30-day overall mortality, occurred in $14 \%$ of patients, but this risk varied according to whether patients were in- or outpatients, had SPE or UPE, and had symptoms and/or abnormal vital signs suggestive of PE. Other adverse cancer-specific and general predictors of increased 
30-day mortality were also identified, including upper gastrointestinal malignancy, disease progression, poor performance status or history of VTE. This suggests that cancer-associated PE is a clinically heterogenic entity that harbours potentially many distinct prognostic sub-phenotypes. The investigators have tried to harness this heterogeneity by defining a novel three-level classification of cancer-associated PE including SPE (30-day mortality 21\%), UPE-S (30-day mortality 20\%) and TA-UPE (30-day mortality 3\%). Importantly, the TA-UPE group accounted for more than $30 \%$ of patients in the study, suggesting that this is a large group of patients who probably may be safely treated in the outpatient setting.

4) Beyond symptoms, the presence of abnormal vital signs (such as systolic blood pressure $<100 \mathrm{mmHg}$, heart rate $>100$ beats per minute and oxygen saturation $<95 \%)$ predicted an increased risk of short-term mortality. Previous studies have arrived at the same conclusion $[7,8]$, suggesting that these clinical signs are the "red flags" to watch out for when managing a patient with cancer-associated PE.

5) Despite the variability in mortality according to symptoms, signs and suspicion, the VTE recurrence rates during anticoagulation were low ( $2 \%$ overall) and did not differ according to investigated baseline risk factors or between the novel classification subgroups. This is another disappointing setback for research on recurrent PE risk stratification in cancer patients [21].

6) While "cancer" or "PE and other conditions" were adjudicated as the cause of death in the vast majority of patients, only $2 \%$ of deaths in EPIPHANY were attributed to PE as the sole cause of death. This strikingly low proportion is consistent with the concept that patients with cancer-associated PE die from their malignancy and associated conditions rather than from fatal PE. Cancer-associated PE thus appears to be more of an epiphenomenon of an aggressive tumour biology and an indicator of very adverse prognosis, and less of a fatal event by itself.

These observational findings are interesting, but do they also inform clinical decision making in initial management and/or anticoagulation? And if so, how? Regarding initial management, current guidelines for the general PE population recommend to use a PE severity assessment tool such as the (s)PESI in non-high risk patients to define the management strategy in the acute setting and support the decision on whether an in- or outpatient treatment approach can be followed [14]. These tools are based on short-term mortality, with a higher predicted risk of short-term mortality indicating that an inpatient management is preferred. FONT et al. [6] have addressed this short-term mortality specifically in the population of cancer patients with PE. Importantly, they defined a novel classification, which shows that patients with a so-called TA-UPE had only a much smaller risk of 30-day mortality of 3\%, whereas all other patients (either presenting with SPE or UPE with symptoms/signs suggestive of PE (UPE-S)) had a 30-day risk of death of around $20 \%$. As TA-UPE patients accounted for more than $30 \%$ of the study population, the proposed subclassification of UPE events suggests that this may be useful for identifying a large proportion of patients with cancer-associated PE who are the right candidates for outpatient management. Nevertheless, the classification has some drawbacks. First, symptoms are highly subjective both from a patient and physician perspective, and different physicians may classify symptoms differently. Second, the classification was derived qualitatively rather quantitatively in a way that is not fully perceivable from their published data. Third, the advantage of a three-group classification is unclear to us when the authors actually clearly showed that only the TA-UPE group had its distinct (low) mortality profile. This criticism, however, should not conceal that the current study is, among other previous work on this issue $[7,8,22]$, a stringent attempt to classify cancer-associated PE. Regarding antithrombotic therapy, two end-points are of interest, namely recurrent VTE and major bleeding [9]. Here, EPIPHANY found a risk of major and fatal bleeding of $4 \%$ and $1 \%$, respectively. However, due to the short follow-up period of 90 days, the relevance of these numbers to long-term anticoagulant treatment of cancer-associated PE is limited. Otherwise, PE recurrence risk was low and could not be predicted with the variables under study. Furthermore, the proposed PE classification could neither identify patients with a higher risk of recurrent PE, nor with a higher risk of bleeding. EPIPHANY also did not generate data on whether anticoagulation may be safely withheld in patients with unsuspected PE and a very low clot burden, which is a much debated issue [20]. The proposed classification will therefore be relevant for questions surrounding the initial management of cancer-associated PE, but not for questions surrounding mid-to-long-term anticoagulation.

In conclusion, this large cohort study by FonT et al. [6] sheds new light on the contemporary clinical course of suspected and unsuspected cancer-associated PE. Within the limitations of an observational study, the authors presented a novel cancer-associated PE risk classification that discriminates between a patient group with UPE without symptom burden (TA-UPE) who experienced a 30-day risk of death of only $3 \%$, and all other patients who experienced 30 -day risk of death of around $20 \%$. In our opinion, this scheme may help physicians to identify patients suitable for outpatient treatment in the cancer setting, similarly to the (s)PESI in the general PE population, when independently validated in other studies. The proposed classification would also be suitable as a stratification factor for future (and much-needed) 
UPE-specific treatment trials. However, the proposed groups of SPE, TA-UPE and UPE-S are relatively cumbersome, and we feel that an easy-to-remember rule based on the absence or presence of symptoms, signs, suspicion and an inpatient or outpatient setting at diagnosis (“4S") would best serve this study's objective on how to assess the severity of cancer-associated PE, apply risk-adjusted management strategies in acute PE and identify cancer patients suitable for outpatient management. However, further controlled studies are definitely needed to independently validate this risk assessment tool and its consequences on PE outcomes.

\section{References}

1 Posch F, Riedl J, Reitter EM, et al. Hypercoagulabilty, venous thromboembolism, and death in patients with cancer. A Multi-State Model. Thromb Haemost 2016; 115: 817-826.

2 van der Hulle T, den Exter PL, Planquette B, et al. Risk of recurrent venous thromboembolism and major hemorrhage in cancer-associated incidental pulmonary embolism among treated and untreated patients: a pooled analysis of 926 patients. J Thromb Haemost 2016; 14: 105-113.

3 Di Nisio M, Lee AY, Carrier M, et al. Diagnosis and treatment of incidental venous thromboembolism in cancer patients: guidance from the SSC of the ISTH. J Thromb Haemost 2015; 13: 880-883.

4 Bleker SM, Beenen LF, Di Nisio M, et al. Incidental pulmonary embolism in cancer patients: Interobserver agreement on the diagnosis and extent with a focus on distal clots. Thromb Res 2016; 147: 46-51.

5 Liebman HA, O'Connell C Incidental venous thromboembolic events in cancer patients: what we know in 2016. Thromb Res 2016; 140, Suppl. 1, S18-S20.

6 Font C, Carmona-Bayonas A, Beato C, et al. Clinical features and short-term outcomes of cancer patients with suspected and unsuspected pulmonary embolism: the EPIPHANY study. Eur Respir J 2017; 49: 1600282

7 Kline JA, Roy PM, Than MP, et al. Derivation and validation of a multivariate model to predict mortality from pulmonary embolism with cancer: The POMPE-C tool. Thromb Res 2012; 129: e194-e199.

8 den Exter PL, Gómez V, Jiménez $\mathrm{D}$, et al. A clinical prognostic model for the identification of low-risk patients with acute symptomatic pulmonary embolism and active cancer. Chest 2013; 143: 138-145.

9 Posch F, Königsbrügge $\mathrm{O}$, Zielinski $\mathrm{C}$, et al. Treatment of venous thromboembolism in patients with cancer: A network meta-analysis comparing efficacy and safety of anticoagulants. Thromb Res 2015; 136: 582-589.

10 Font C, Farrús B, Vidal L, et al. Incidental versus symptomatic venous thrombosis in cancer: a prospective observational study of 340 consecutive patients. Ann Oncol 2011; 22: 2101-2106.

11 den Exter PL, Hooijer J, Dekkers OM, et al. Risk of recurrent venous thromboembolism and mortality in patients with cancer incidentally diagnosed with pulmonary embolism: a comparison with symptomatic patients. J Clin Oncol 2011; 29: 2405-2409.

12 Lee AY, Kamphuisen PW, Meyer G, et al. Tinzaparin $v s$ warfarin for treatment of acute venous thromboembolism in patients with active cancer: a randomized clinical trial. JAMA 2015; 314: 677-686.

13 Donzé J, Le Gal G, Fine MJ, et al. Prospective validation of the Pulmonary Embolism Severity Index. A clinical prognostic model for pulmonary embolism. Thromb Haemost 2008; 100: 943-948.

14 Konstantinides SV. 2014 ESC Guidelines on the diagnosis and management of acute pulmonary embolism. Eur Heart J 2014; 35: 3145-3146.

15 Jiménez D, Aujesky D, Moores L, et al. Simplification of the pulmonary embolism severity index for prognostication in patients with acute symptomatic pulmonary embolism. Arch Intern Med 2010; 170: 1383-1389.

16 Font C, Carmona-Bayonas A, Fernández-Martinez A, et al. Outpatient management of pulmonary embolism in cancer: data on a prospective cohort of 138 consecutive patients. J Natl Compr Canc Netw 2014; 12: 365-373.

17 Carmona-Bayonas A, Font C, Jiménez-Fonseca P, et al. On the necessity of new decision-making methods for cancer-associated, symptomatic, pulmonary embolism. Thromb Res 2016; 143: 76-85.

18 Moores LK, Zamarro C, Gómez V, et al. Early discharge and outpatient treatment of patients admitted for acute pulmonary embolism. Eur Respir J 2013; 41: 487-488.

19 Peris M, Jiménez D, Maestre A, et al. Outcome during and after anticoagulant therapy in cancer patients with incidentally found pulmonary embolism. Eur Respir J 2016; 48: 1360-1368.

20 Yoo HH, Queluz TH, El Dib R. Anticoagulant treatment for subsegmental pulmonary embolism. Cochrane Database Syst Rev 2014; CD010222.

21 Ahn S, Lim KS, Lee YS, et al. Validation of the clinical prediction rule for recurrent venous thromboembolism in cancer patients: the Ottawa score. Support Care Cancer 2013; 21: 2309-2313.

22 O'Connell CL, Razavi PA, Liebman HA. Symptoms adversely impact survival among patients with cancer and unsuspected pulmonary embolism. J Clin Oncol 2011; 29: 4208-4209. 